\title{
Enfermedad tromboembólica venosa en poblaciones específicas: mucho por aprender
}

Lucía Ordieres-Ortega, Pablo Demelo-Rodríguez

Hospital General Universitario Gregorio Marañón

La enfermedad tromboembólica venosa (ETV), que incluye principalmente el embolismo pulmonar (EP) y la trombosis venosa profunda (TVP) de extremidades inferiores, es una causa importante de mortalidad, especialmente en grupos poblacionales concretos como los pacientes oncológicos o la mujer embarazada. La evidencia disponible sobre el tratamiento y la evolución de la ETV en determinadas poblaciones es escasa. En el presente número de Galicia Clínica, dos artículos van a abordar aspectos interesantes de la ETV en el embarazo y en pacientes con enfermedades autoinmunes sistémicas.

El embarazo supone un aumento fisiológico, ya conocido y ampliamente estudiado, del riesgo trombótico en la mujer'. Como expone el artículo por Díaz Peromingo et al, el embarazo produce un estado de hipercoagulabilidad como preparación previa al parto. Este incremento del riesgo protrombótico se mantiene, incluso de manera más pronunciada, en el postparto inmediato.

Los signos y síntomas habituales de la ETV, así como marcadores normalmente útiles para el despistaje, como el dímero D, son inespecíficos en la mujer embarazada, por lo que el diagnóstico supone un desafío en sí mismo ${ }^{3}$. Recientemente, el algoritmo YEARS fue validado como herramienta para excluir la EP en embarazadas². En caso de existir sospecha clínica de ETV, se debe confirmar el diagnóstico con técnicas de imagen adecuadas.

En este artículo, los autores repasan la evidencia disponible y las indicaciones de profilaxis antitrombótica en el embarazo, incluyendo asimismo un tema complejo como son las trombofilias y la problemática del manejo específico en estas pacientes. El mayor riesgo de ETV se ha descrito en pacientes con factor $V$ Leiden en homocigosis o la mutación para el gen de la protrombina G20210A4, y se recomienda valorar profilaxis antitrombótica en pacientes embarazadas con trombofilia y antecedentes personales de ETV. La escala de Lyon ayuda a valorar la necesidad de profilaxis antitrombótica, estableciendo el riesgo de ETV durante el embarazo².
Díaz Peromingo et a $\mathbb{R}^{\mathbb{2}}$ realizan además una revisión de las indicaciones del filtro de vena cava (FVC) en la mujer embarazada. El FVC es un dispositivo de utilización en aumento en las últimas décadas, a pesar de no haberse evidenciado una reducción de la mortalidad asociada a la ETV5. Su principal indicación (y la única con evidencia suficiente) es el tratamiento en pacientes con ETV aguda y contraindicación absoluta para la anticoagulación. Se dispone de FVC permanentes o transitorios, recomendándose utilizar estos últimos con retirada precoz siempre que sea posible.

En el caso de las pacientes embarazadas con ETV, se postula que podría existir un subgrupo de pacientes con alto riesgo de recurrencia trombótica a pesar de anticoagulación correcta, en las que se podría plantear la colocación de un FVC de cara al parto, con retirada precoz $z^{2,3}$. Sin embargo, se han descrito complicaciones como la perforación de la vena cava o la migración del filtro como consecuencia de la dilatación de la vena cava durante el embarazo ${ }^{5}$, por lo que se deben valorar cuidadosamente los riesgos y beneficios del procedimiento en cada caso individual.

Es necesaria más evidencia de cara a establecer un adecuado manejo en las mujeres embarazadas con ETV, especialmente al enfrentarse a problemas tan complejos como la profilaxis antitrombótica en mujeres embarazadas con trombofilias o las indicaciones de los FVC en los momentos del preparto y del postparto.

En el segundo artículo, Enfermedad Tromboembólica Venosa en Pacientes con Enfermedades Autoinmunes en el Hospital Reina Sofía de Tudela ${ }^{6}$, los autores buscan extrapolar a su población los hallazgos de un artículo del Registro Informatizado de Enfermedad Tromboembólica ${ }^{7}$ (RIETE), que establece que los pacientes con enfermedades autoinmunes sistémicas (EAS) presentan mayor riesgo de ETV y mayor mortalidad que aquellos pacientes sin EAS, pero no un aumento de las recurrencias o hemorragias mayores ${ }^{8}$.

Para ello, los autores recogieron información de 222 pacientes, de los cuales 19 presentaban una 
Es necesaria más evidencia de cara a establecer un adecuado manejo en las mujeres embarazadas con ETV

enfermedad autoinmune sistémica, y realizaron un seguimiento mínimo de 10 años. El riesgo de recurrencia de ETV en los pacientes con EAS fue mayor, pero no se encontró una mayor mortalidad o un aumento del riesgo de hemorragia mayor. Como destacan los autores, es llamativa la alta prevalencia de EAS en su población (8.55\%), así como una menor presencia de enfermedad renal y de cáncer respecto al estudio de RIETE, lo cual podría influir en la aparición de ETV, y no se recogen otros factores como el uso de medicaciones.

Se ha descrito un mayor riesgo de ETV en pacientes con EAS, especialmente durante los episodios de enfermedad activa y en algunas entidades específicas como la artritis reumatoide (riesgo mayor del doble $)^{9}$, si bien las razones no están completamente claras $^{10}$. El papel del tratamiento inmunosupresor ha sido investigado en la enfermedad de Behçet, pero no en otras, y el rol de la anticoagulación aún debe ser estudiado, no siendo posible realizar recomendaciones generales para el tratamiento de estos pacientes ${ }^{10}$.

La enfermedad tromboembólica venosa es una patología muy frecuente y cuya frecuencia está en aumento. Aunque la evidencia disponible sobre el manejo y pronóstico de la enfermedad es cada vez mayor, existen en la actualidad importantes lagunas de conocimiento, fundamentalmente en lo que respecta a grupos poblacionales más pequeños como embarazadas, pacientes con enfermedades autoinmunes, pacientes con trombofilia, etc. En estas poblaciones en las que la realización de ensayos clínicos es más compleja, cobran especial interés los datos surgidos de estudios poblaciones y de vida real, que aportan información relevante y abren la puerta al diseño de nuevos estudios.

\section{REFERENCIAS}

1. Bates SM. Pregnancy-associated venous thromboembolism: prevention and treatment. SeminHematol. 2011;48(4):271-84.

2. Díaz Peromingo JA, Núñez Fernández MJ. Filtro de vena cava y embarazo: algunas certezas y muchas incertidumbres. Galicia Clin 2021; 82-4: 208 - 213.

3. McLintockC, Brighton T, Chunilal S, Dekker G, McDonnell N, McRae Set al. Recommendations for the diagnosis and treatment of deep venousthrombosis and pulmonary embolism in pregnancy and the postpartumperiod. Aust N Z J ObstetGynaecol. 2012;52(1):14-22.

4. Lussana F, Coppens M, Cattaneo M, Middeldorp S. Pregnancy-related venous thromboembolism: risk and the effect of thromboprophylaxis. Thromb Res. 2012;129(6):673-80.

5. Duffet L, Carrier M. Inferior Vena Cava filters. J ThrombHaemost. 2017;15:3-12.

6. Ruiz-Sada P, Sampériz-Legarre A, Galbete A. Enfermedad Tromboembólica Venosa en Pacientes con Enfermedades Autoinmunes en el Hospital Reina Sofía de Tudela. [Resto de referencia].

7. Bikdeli B, Jimenez D, Hawkins M,Ortíz S, Prandoni P, Brenner B et al. Rationale, Design and Methodology of the Computerized Registry of Patients with Venous Thromboembolism (RIETE). ThrombHaemost. 2018;118(1):214-224.

8. Ruiz Sada P, López-Núñez JJ, Samperiz A, Soto MJ, Pedrajas JM, Porras JA et al. Venous Thromboembolism in Patients With Autoimmune Disorders: Findings From the RIETE Registry. Angiology. 2020;71(2):131-138.

9. Kefti C, Boutigny A, Mohamedi N, Bouajil S, Magnan B, Amah G et al. Risk of venous thromboembolism in rheumatoid arthritis. Joint Bone Spine. 2021;88(3):105122.

10. Tamaki $H$, Khasnis A. Venous thromboembolism in systemic autoimmune diseases: a narrative review with emphasis on primary systemic vasculitides. Vasc Med. 2015;20(4):369-76. 